\title{
Characteristics of GMR-SV Sensor for Measurement of Mineral Contents in Edible Water
}

\author{
Da-Woon Kim ${ }^{1}$, Ju-Hee Lee ${ }^{1}$, Min-Ji Kim ${ }^{1}$, and Sang-Suk Lee ${ }^{2 *}$ \\ ${ }^{1}$ Kangwon Science High School, Wonju-City, Gangwon-do, Korea \\ ${ }^{2}$ Dept. of Oriental Biomedical Engineering, Sangji University, Wonju-City, Gangwon-do, Korea
}

(Received 15 January 2009, Received in final form 1 April 2009, Accepted 3 April 2009)

\begin{abstract}
The mineral dissolution sensor system using GMR-SV and glass/Mg(200 $\mathrm{nm})$ was prepared and characterized. The magnetic field sensitivity of GMR-SV to microscopic magnetic variation was about $0.8 \% / O e$. The change that occurs when Mg-film dissolves in water, the solubility of water, which is one of the basic properties of mineral water, was sensed by measuring the subtle variation of an electric current. In the case of edible water with Mg mineral added, bubbles were generated on the surface of the Mg film in the first $\mathbf{4 5}$ minutes, and the number of drops that were dissolved more rapidly than with the tap and DI waters later reduced to zero. For the edible water samples that each had different mineral Mg concentrations, the Mg solubility speed significantly differed. After injecting Mg film into the edible water, the magnetoresistance of the output GMR-SV signal decreased from a maximum of $45.4 \Omega$ to a minimum of 43.6 $\Omega$ The measurement time was within 1 min, giving the rate of change $\Delta R / \Delta t=0.18 \Omega / \mathrm{s}$. This measurement system can be applied to develop a mineral Mg solubility GMR-SV sensor that can be used to sense the change from edible water to reduced alkali.
\end{abstract}

Keywords : GMR-SV (giant magnetoresistance-spin valve), Mg-film, Mineral edible water, ORP (oxidation reduction potential)

\section{Introduction}

The fertilized egg, which forms the beginning of the human body, consists of $99 \%$ water, while the newly born human body comprises $90 \%$ water. When a human is fully grown, due to the increasing percentage of mineral content substances in bones, the percentage of water forming the body will decrease to $70 \%$ and will further decrease to $50 \% \sim 60 \%$ before death. Because water is such an essential component in human lives water depletion can be fatal, thus the phrase, 'water is life'. Therefore, we can become healthier by increasing our water content from $70 \%$ to $90 \%$, the water content of a new born, according to how we control the water we drink [1, 2].

Electrolysis alkali water is as effective as vitamin $\mathrm{C}$ or vitamin $\mathrm{E}$ in eliminating active oxygen. The presence of active oxygen in the human body is caused by stress, alcohol, smoking, pollution, processed food, and retrogressive diseases such as cancer or circulatory system di-

*Corresponding author: Tel: +82-33-738-7961

Fax: +82-33-738-7962, e-mail: sslee@sangji.ac.kr seases such as disease that attack lipid cells. Active oxygen is known to be removed by vitamin $\mathrm{C}$ and vitamin $\mathrm{E}$, which are supplied to the body through what we eat. The amount of dissolved oxygen in electrolysis alkali water does not differ from that in tap water $(10 \mathrm{ppm} \rightarrow 8 \sim 9$ ppm), but the amount of hydrogen molecules in alkali water was hundreds of times more abundant than it was in tap water ( $2 \mathrm{ppb} \rightarrow 400 \sim 500 \mathrm{ppb}$ ). According to a recently announced report, the active hydrogen (hydrogen atom) found in electrolysis alkali water is able to remove active oxygen [3,4]. Actually, the ability to remove active oxygen disappeared when three oxide tungsten (tungsten is capable of selectively absorbing only active hydrogen), were placed in the device. This suggests that the ability to eliminate active oxygen depends not only on hydrogen molecules $\left(\mathrm{H}_{2}\right)$ but also on active hydrogen $(\mathrm{H} \cdot)$ balanced with a hydrogen molecule. Also, the ability of electrolysis alkali water to exclude active oxygen is considerably stable, and it can even be maintained for more than one month at a condition of $4{ }^{\circ} \mathrm{C}$. Although the high pressured pasteurization or penetration was repeatedly performed by the filter using the pistons in the experiment, the ability to exclude active oxygen did not lessen. However, active 
hydrogen easily reacts with oxygen because active hydrogen has a superior reaction property. It is difficult for dissolved oxygen in electrolysis alkali water and hydrogen atoms to exist in a stable condition without reacting with each other for more than one month in a general environment [5].

The typical alkali water manufacturing system that is usually used performs electrolysis of water using direct current electricity. However, there are other methods besides using electrolysis of water. One of the simplest methods is using inorganic minerals. This method is very inexpensive and portable, and provides a good edible water taste. Certain minerals change water into alkali while emitting hydrogen as they contact and decompose. These minerals are $\mathrm{Mg}, \mathrm{Ca}$, and $\mathrm{Li}$, which have a greater tendency for ionization than hydrogen. The mineral components of $\mathrm{Ca}$ and $\mathrm{Li}$ have good character, creating a close water structure. However, these minerals cannot be used in edible water since they react violently with water. On the other hand, $\mathrm{Mg}$ ion can be used for alkali reduction water because it reacts slowly with water [6].

Because of its convenience in manufacture and analysis and superior discernment ability, a highly sensitive nano magnetic element could be applied to sensors in the fields of engineering, optics, biochemistry, life science, and for diagnosis in medical science. In a low magnetic field, highly sensitive nano magnetic elements show a high signal to noise ratio $(\mathrm{S} / \mathrm{N})$ output and various characteristics such as a highly sensitive degree of magnetoresistance. Those can be developed into commercial items such as minute chips of a living body molecule which is highly accumulated. If a minute nano magnetic element can be manufactured and its characteristics can be reproduced, it will become an extremely important element for the future of the nano-biology industry, for the future of bioengineering technology and in the medical treatment industry. The new element that will contribute to the development of an advanced national industry is important in terms of the development of the nano-biosensor technique [7-9]. Korea could become an advanced science nation having this ultra tiny magnetoresistance film element manufacture technique and research or application based on a semiconductor accumulation circuit technique.

In this study, an $\mathrm{Mg}$ sensor element was designed and fabricated using a giant magneoresistance-spin valve (GMR-SV) sensor to observe the characteristics of Mgfilm of $200 \mathrm{~nm}$ thickness dissolved in water. In the microscopic view of the electron which offers power of reduction through making hydrogen for exclusion activity oxygen in mineral water, $\mathrm{Mg}$-film dissolves in nano size. The ability to sense mineral dissolution, which is one of the basic properties in developing mineral reduction water, was developed by measuring the tiny change in an electric current flowing through the Mg-film.

\section{Experimental Method}

The operating system used has a sensor that perceives the ability of minerals to reduce alkali in water by using a highly sensitive GMR-SV device with a multilayer type, as shown in Fig. 1. The main reservoir for $\mathrm{Mg}$-film, $\mathrm{Cu}-$ foil, and $\mathrm{Cu}$-electrode was made of an acryl and could contain up to $20 \mathrm{cc}$ of water. The $\mathrm{Mg}$-film was placed on the center of the slide glass and was wrapped with the $\mathrm{Cu}$-foil. This sample was then positioned on the stand and made able to contact the $\mathrm{Cu}$ electrode by driving a screw. $\mathrm{Mg}$-film is prepared from sublimation film placed in a thermal evaporation system at nearly $1000^{\circ} \mathrm{C}$ and then formed into a strip using a shadow mask. Using a $6 \mathrm{MHz}$ quartz crystal oscillator, the Mg-film was measured to $200 \mathrm{~nm}$. Our operation system uses a solenoid made with a coil (diameter is $0.5 \mathrm{~mm}$ ) rolled 200 times to maintain the equalized magnetic field at $10 \mathrm{Oe}$, the linearity of which is perceived by the GMR-SV.

The measured waters are edible, tap, and DI water, the content of which is known to be $\mathrm{Mg}, \mathrm{K}, \mathrm{Na}$, and $\mathrm{Ca}$. The inorganic contents of the edible water are; $\mathrm{Ca}=5.8 \sim 34.1$ $\mathrm{mg} / \mathrm{l}, \mathrm{Na}=2.5 \sim 10.7 \mathrm{mg} / \mathrm{l}, \mathrm{K}=0.3 \sim 1.4 \mathrm{mg} / \mathrm{l}, \mathrm{Mg}=0.8 \sim 5.4$ $\mathrm{mg} / \mathrm{l}$, and $\mathrm{F}=0 \sim 1.2 \mathrm{mg} / \mathrm{l}$. The oxidation reduction potential (ORP) of water was measured with a standard device while $\mathrm{Mg}$ shots (diameter is 2 3 mm) were dissolved [10,

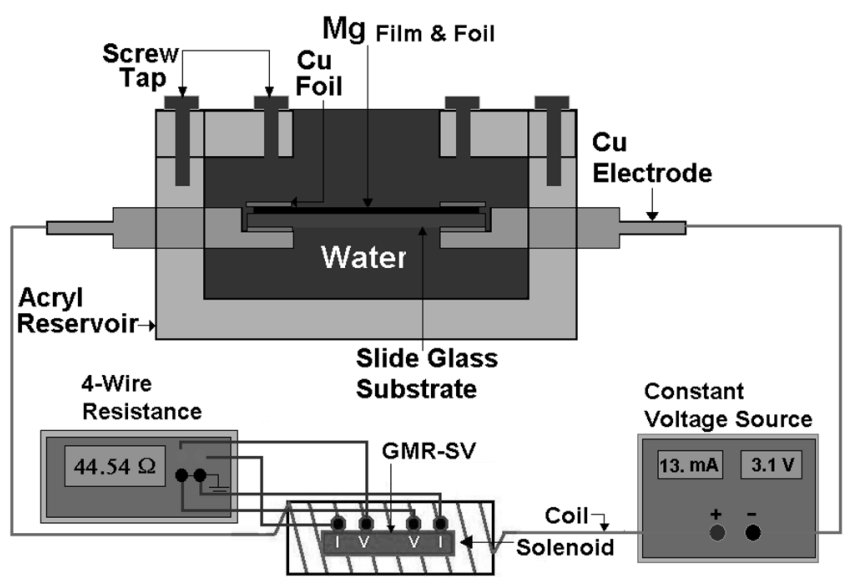

Fig. 1. Schematic of mineral alkali reducing water sensor system using a highly sensitive GMR-SV device with a multilayer type. Two materials of dissolved $\mathrm{Mg}$ are film and foil inside water in $20 \mathrm{cc}$ volume acryl reservoir. $\mathrm{Cu}$ tape is used for the electrical contact between the electrode and Mg-material. The characteristics of the GMR-SV device are measured by a 4wire resistance that is set inside the solenoid, which is supplied by constant source meter and voltage source. 
(a)

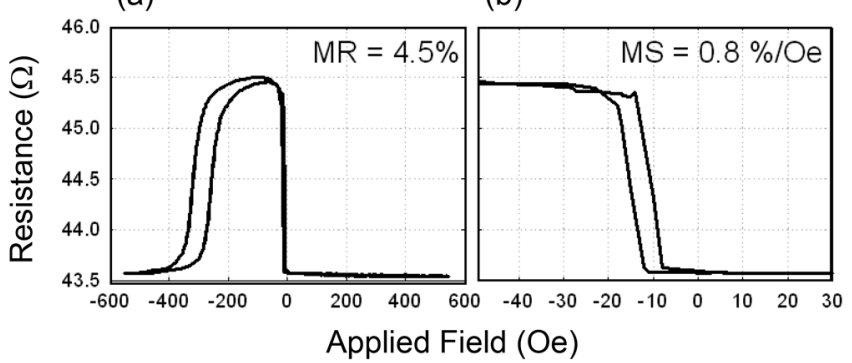

Fig. 2. MR (a) major and (b) minor loop for the GMR-SV with $\mathrm{Sub} . / \mathrm{Ta}(5 \mathrm{~nm}) / \mathrm{NiFe}(7 \mathrm{~nm}) / \mathrm{Cu}(2.5 \mathrm{~nm}) / \mathrm{NiFe}(5 \mathrm{~nm}) /$ $\mathrm{FeMn}(12 \mathrm{~nm}) / \mathrm{Ta}(5 \mathrm{~nm})$ multilayer, which is prepared by the $\mathrm{dc}$ magnetron sputtering system. The measured values of MR ratio and 4-wire resistance are $4.5 \%$ and $43.5 \Omega$, respectively. The exchange coupling field and coercivity of the pinned $\mathrm{NiFe}$ layer are 300 Oe and 35 Oe, respectively. The magnetic sensitivity is $0.8 \% / \mathrm{Oe}$ at about 10 Oe.

11]. The dissolved $\mathrm{Mg}$ is measured by the variation of the amount of bubbles with an optical real microscope.

\section{Experimental Results and Discussion}

The major MR curve and minor MR curve are shown in Figs. 2(a) and 2(b). Those MR curves are obtained by the GMR-SV device having a feature that searches for the dissolving Mg-film. Multilayer with $\mathrm{Ta}(5) / \mathrm{NiFe}(7) / \mathrm{Cu}(2.5)$ $/ \mathrm{NiFe}(5) / \mathrm{FeMn}(8)(\mathrm{nm})$ bottom type is deposited in a 4arget dc magnetron sputtering system, and the working pressure during deposition is maintained at 2.5 mTorr [12]. The maximum MR ratio is $4.5 \%$, and the 4-wire resistance is about $43.5 \Omega$ The commutative coupling field intensity and coercivity of the pinned $\mathrm{NiFe}$ layer are $300 \mathrm{Oe}$ and $35 \mathrm{Oe}$, respectively. Also, the value of the applied current to the solenoid and the corresponding magnetic field required to obtain an output signal of GMR-SV according to Mg solubility are $1 \mathrm{~mA}$ and $1 \mathrm{Oe}$, respectively. Due to the interaction between the bottom magnetic free layer and the top magnetic pinned layer, the curve's center shifted at about -13 Oe, and the coercivity was about $1.5 \mathrm{Oe}$. The magnetic sensitivity, which is very sensitive at a subtle variation of the outer magnetic field, was about $0.8 \% / \mathrm{Oe}$ at $-10 \mathrm{Oe}$ [13].

For mineral alkali water, the $\mathrm{Mg}$ content is more important than the Ca content. $\mathrm{Mg}$ can be used as a sensor for dissolving the mineral because $\mathrm{Mg}$ disintegrates water when it dissolves in water and releases an electron, following the chemical formula of (1) [14].

$$
\mathrm{Mg}+2 \mathrm{H}_{2} \mathrm{O} \rightarrow \mathrm{Mg}^{2+}+2 \mathrm{OH}^{-}+\mathrm{H}_{2}\left(\mathrm{H}^{\cdot}+\mathrm{H}^{\cdot}=\mathrm{H}_{2}\right)
$$

Because $\mathrm{OH}-$ is formed by this dissolving process, water

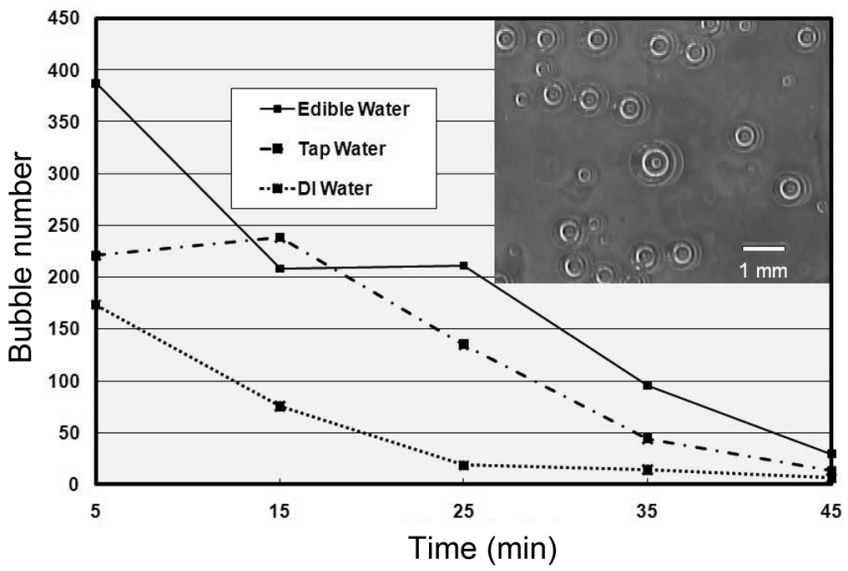

Fig. 3. The variation of $\mathrm{Mg}$-bubble number as a function of time for three different waters; edible, tap, and distilled water. The typical feature of a series of Mg-bubbles is shown in the inset at the right top side. The rates at which $\mathrm{Mg}$ dissolved after 45 min are shown the order of edible $>$ tap $>$ DI water.

is alkalified. Simultaneously, $\mathrm{H}_{2}$ and active hydrogen that are formed by the dissolving process make the coater able to remove active oxygen as they decrease the ORP of water. Compared to $\mathrm{Ca}, \mathrm{Mg}$ 's ability to remove active oxygen is relatively weak [15]. Supplementary materials can be used to treat the problem; ceramics that slowly release $\mathrm{Ca}$, special minerals that naturally ensure the stability of active hydrogen, rocks, which help the water to dissolve easily and create a pleasant water structure, and minerals in rocks that act as catalysts.

$\mathrm{Mg}$ film forms bubbles during the dissolving process in $20{ }^{\circ} \mathrm{C}$ water. The typical shape of the bubbles is indicated in the upper right side of Fig. 3. It is thought that $\mathrm{H}_{2}$ is formed from two active hydrogen bonds that unite during the dissolving process, and the amount of bubbles depends on the inorganic substance content of water and on the solubility of Mg. Fig. 3 indicates the variation in the numbers of Mg-bubbles as a function of time for the three kinds of water; edible, tap, and distilled. In the case of distilled water that contains a small quantity of inorganic $\mathrm{Mg}$, not only are there fewer bubbles, but after 45 min the $\mathrm{Mg}$ film is more dissolved than it is in edible and tap water. It is considered to be an effect that depends on the inorganic substance content and variation of $\mathrm{pH}$.

Fig. 4 shows variations of ORP with the lapse of time for $200 \mathrm{cc}$ of edible water at a constant $20{ }^{\circ} \mathrm{C}$ with $\mathrm{Mg}$ arranged to melt uniformly. The times from acidity into reducing alkali required for the changing ORP of three different water types from positive to negative are $40 \mathrm{~min}$, $38 \mathrm{~min}$, and $20 \mathrm{~min}$, respectively. The extents of change of ORP with the lapse of time for the three types of water are $27 \mathrm{mV}, 27 \mathrm{mV}$ and $10 \mathrm{mV}$, respectively. For edible 


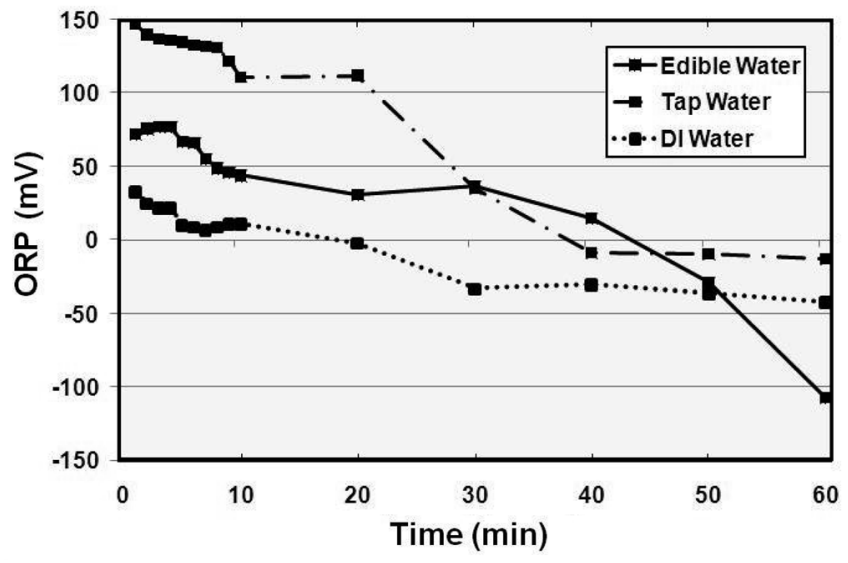

Fig. 4. The variation of ORP (oxidation reduction potential) as a function of time for three different waters; edible, tap, and distilled water. The change times of reduced alkali for edible, tap, and distilled water are $43 \mathrm{~min}, 38 \mathrm{~min}$, and $20 \mathrm{~min}$, respectively.

and tap water, the point of changing the actually reduced alkali will differ as the initial ORP differs but the variations of ORP are the same because the mineral amount has already melted in the water. Consequently, the solubility of $\mathrm{Mg}$ for the three types of water differs. There is a relationship between ORP variations and changes of the reducing alkali removing active oxygen.

Fig. 5 shows the data obtained from the measurement system shown in Fig. 1. The $\mathrm{X}$ - axis refers to time, and the $\mathrm{Y}$-axis refers to the output signal of magnetic resistance displacement. The sensing current of two electric currents is $1 \mathrm{~mA}$ in four terminal measurements. This is the result from the output of the electric measurement of two terminals. Fig. 5 presents the maximum and minimum step waves between positive and negative through variations of the outside magnetic field. Fig. 5(a) shows that the loop started at +50 Oe and obtained a magnetic resistance of $43.6 \Omega$, passed $0 \mathrm{Oe}$, and ended at $-10 \mathrm{Oe}$. It almost reached -12 Oe, then increased until -20 Oe. Fig. 5(a) are marked about stage of $1,2,3$, and 4 corresponding to hysteresis of MR curve, as shown in Fig. 5(b). When restarting from stage 3 of -30 Oe, the sensitive magnetic point to stage 4 of -10 Oe. In stage 4 , when the magnetic sensitivity is $0.8 \% / \mathrm{Oe}$, the linear shape suggests a variation of one minute outside the magnetic field. In Fig. 1, the resistance of the Mg-film melt increased slowly from $1.5 \Omega$, but the resistance of the solenoid stayed at $5 \Omega$. Therefore, the electric current from the electric pressure provided in $3.0 \mathrm{~V}$ slightly decreased the linear MR curve.

Fig. 6 shows the data for edible water type B of company $\mathrm{H}$, for which $0.8 \sim 54 \mathrm{mg} / \mathrm{l}$ of dissolved $\mathrm{Mg}$ was (a)

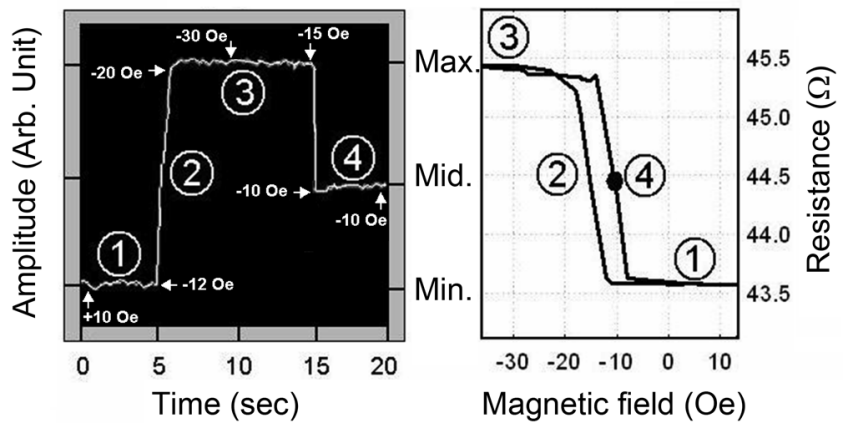

Fig. 5. (a) Step type output signal and (b) MR minor loop for the GMR-SV with $\mathrm{Sub} . / \mathrm{Ta}(5 \mathrm{~nm}) / \mathrm{NiFe}(7 \mathrm{~nm}) / \mathrm{Cu}(2.5 \mathrm{~nm}) /$ $\mathrm{NiFe}(5 \mathrm{~nm}) / \mathrm{FeMn}(12 \mathrm{~nm}) / \mathrm{Ta}(5 \mathrm{~nm})$ multilayer. Here the step numbers of 1, 2, 3, and 4 correspond between (a) and (b). The corresponding magnetic fields for four steps are indicated by arrows. The sensing position and magnetic sensitivity are step 4 with $44.4 \Omega$ and $0.8 \% /$ Oe at about -10.1 Oe, respectively. After complete dissolving of Mg-film in water, the applying current of the solenoid decreased to zero. The resistance of the GMR-SV at zero magnetic field then returned to the original starting point by the final output 4-wire resistance signal of $43.6 \Omega$.

measured using the measuring system shown in Fig. 1. The water tank was covered with an Mg-film of $20 \mathrm{~mm}$ length, $0.5 \mathrm{~mm}$ width, and a thickness of $200 \mathrm{~nm}$. Fig. 6 shows the $\mathrm{x}$-axis as time and the $\mathrm{y}$-axis as output signals. Before putting the $\mathrm{Mg}$ into the edible water type $\mathrm{B}$, measurement was started at a magnetic resistance of 44.5 $\Omega$ as explained in Fig. 5, which is the $4^{\text {th }}$ stage point with the highest magnetic field sensitivity. This indicated a change of magnetic resistance as shown in Fig. 6 where edible and tap water were within 17 and 18 minutes, respectively, the magnetic resistance of the output signal fell to a minimum of $43.6 \Omega$ and the change rates of $\mathrm{Mg}$ solubility for time $\Delta R / \Delta t=0.18 \Omega / \mathrm{s}$, that is, the result when sensing a current of $1 \mathrm{~mA}$ was sent to the GMR-SV device, because $1.8 \mathrm{mV}$ can be measured and can therefore be used as a sensitivity device to perceive the $\mathrm{Mg}$ content.

Now, the nano-bio device using GMR-SV with a convincing bio-recognition system and physical chemistry shows a useful observation of motion of biological and chemical molecules that choose special molecules [16]. As previously seen in Figs. 3 and 4, each type of water dissolves different amounts of minerals. Fig. 6 distinguishes between dissolving time and dissolving speed of Mg-film. This result shows the possibility for the ORP measurement sensor and the $\mathrm{Mg}$ solving sensor. Further research will report on the application and manufacture of mineral reducing measuring sensors shaped as lab-on- 


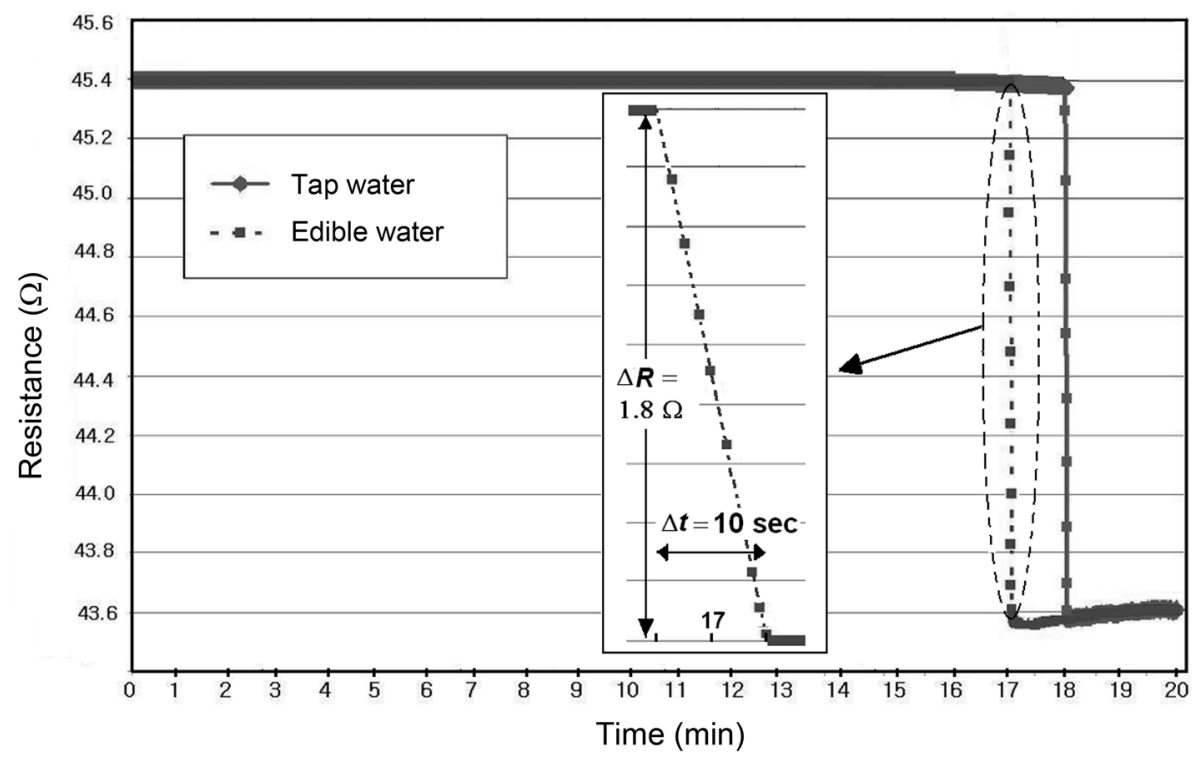

Fig. 6. The variation of output magnetoresistance as a function of dissolved time of Mg-film for edible and tap water, which are composed of a mineral content of $0.8 \sim 5.4 \mathrm{mg} / \mathrm{l}$. The response times for the dissolution of $\mathrm{Mg}$-film with edible and tap water are 17 min and $18 \mathrm{~min}$, respectively. The calculated output resistance signals from the dissolution speed of $\mathrm{Mg}$-film with edible and tap water are $\Delta R / \Delta t=0.18 \Omega / \mathrm{s}$, respectively. The inset shows the slope of output magnetoresistance having the Mg dissolution rate.

chip taking advantage of the nano-magnetic device which is used in bio-health.

\section{Conclusions}

The dissolution sensor system was developed with 200 $\mathrm{nm}$ thick Mg-film and $50 \mu \mathrm{m}$ thick Mg-foil using a GMR-SV device with a magnetic sensitivity of $0.8 \% / \mathrm{Oe}$. During the dissolving process of $\mathrm{Mg}$-film in water, a subtle variation of the magnetic field due to the decrease of current in the solenoid was detected by the GMR-SV sensor. The variations of the numbers of $\mathrm{Mg}$ bubbles and ORP as a function of time for three different waters (edible, tap, and distilled water), were measured and compared with data of other samples. The speed of dissolution of $\mathrm{Mg}$ after $45 \mathrm{~min}$ was in the order of edible $>\operatorname{tap}>$ DI water. The variation of output magnetoresistance of Mg-film for edible water, which is composed of a mineral content of $0.8 \sim 5.4 \mathrm{mg} / \mathrm{l}$, was investigated as a function of dissolved time. The response times for the dissolution of $\mathrm{Mg}$-film with edible and tap water were 17 $\mathrm{min}$ and $18 \mathrm{~min}$, respectively. The rate of change of $\mathrm{Mg}$ solubility was $\Delta R / \Delta t=0.18 \Omega / \mathrm{s}$. When a sensing current of $1 \mathrm{~mA}$ was sent to the GMR-SV device, the output signal could be measured up to $1.8 \mathrm{mV}$. Since the dissolving time and speed for $\mathrm{Mg}$-film in edible water depends on the concentration of $\mathrm{Mg}$ ion, it seems possible that the $\mathrm{Mg}$ mineral sensor could be developed by using the GMR-SV device.

\section{Acknowledgements}

This work was supported in part by the Korea Foundation for International Cooperation of Science \& Technology (KICOS K20702000015-07E0200-0510), by a grant from the Science High School Research \& Education program of KOSEF (2008), and the Sangji University Research Fund, 2007-2008.

\section{References}

[1] D. H. Lee, Physics and Technology (KPS) February, 3 (2003).

[2] L. Frank, J. B. Sigwarth, and J. D. Craven, Geophys. Res. Lett. 12, 303 (1986).

[3] A. J. Swallow, Nature 222, 369 (1969).

[4] M. Armbruster, H. Haberland, and H. G. Schinder, Phys. Rev. Lett. 47, 323 (1981).

[5] S. Shirahata, S. Kabayama, M. Nakano, et al., Biochem. Biophys. Res. Commun. 234, 269 (1981).

[6] Y. Tanaka, K. Kikuchi, Y. Saihara, and Z. Ogumi, Electrochimica Acta 50, 5229 (2005).

[7] D. R. Baselt, G. U. Lrr, M. Natesan, S. W. Metzger, P. E. Sheehan, and R. J. Colton, Biosens. \& Bioelec. 13, 731 (1998).

[8] J. S. In, S. H. Kim, J. Y. Kang, A. Tiwari, and J. I. Hong, J. Magnetics 12, 118 (2007).

[9] D. R. Baselt, G. U. Lee, and R. J. Colton, J. Vac. Sci. Technol. B 14, 789 (1996).

[10] J. Vilcáez, K. Suto, and C. Inoue, Inter. J. Mineral Pro- 
cessing 88, 37 (2008).

[11] M. Banhidi, Metal Finishing 105, 518 (2007).

[12] S. K. Kim, S. C. Shin, and K. Y. Kim, J. Kor. Phys. Soc. 39, 1060 (2001).

[13] M. C. Ahn, S. D. Choi, H. W. Joo, G. W. Kim, D. G. Hwang, J. R. Rhee, and S. S. Lee, J. Kor. Magn. 17, 156 (2007).
[14] F. A. Cotton, G. Wilkinson, and P. L. Gauss, "Basic Iorganic Chemistry", Third Edition, John Wiley \& Sons, Inc., Chapter 9 (1996).

[15] G. Du, B. Wang, Earth Science Frontiers 15, 142 (2008).

[16] S. H. Park, K. S. Soh, D. G. Hwang, J. R. Rhee, and S. S. Lee, J. Magnetics. 13, 30 (2008). 